\title{
Birth weight and the risk of atrial fibrillation in whites and African Americans: the Atherosclerosis Risk In Communities (ARIC) study
}

\author{
Sherifat O Lawani ${ }^{1}$, Ellen W Demerath ${ }^{1}$, Faye L Lopez ${ }^{1}$, Elsayed Z Soliman², Rachel R Huxley ${ }^{3}$, Kathryn M Rose ${ }^{4}$
} and Alvaro Alonso ${ }^{1 *}$

\begin{abstract}
Background: Low birth weight (LBW) has been associated with an increased risk of cardiovascular disease (CVD). A previous study, however, found higher risk of atrial fibrillation (AF) in individuals with higher birth weight (BW). To further understand this apparent paradox, we examined the relationship between AF and BW in the Atherosclerosis Risk in Communities (ARIC) cohort.

Methods: The analysis included 10,132 individuals free of AF at baseline (1996-1998), who provided BW information, were not born premature, and were not a twin. Self-reported BW was categorized as low $(<2.5 \mathrm{~kg})$, medium $(2.5-4 \mathrm{~kg})$, and high $(>4.0 \mathrm{~kg}$ ). AF incidence was ascertained from hospital discharge codes and death certificates. We used multivariable Cox proportional hazard models to determine the hazard ratios (HR) and 95\% confidence intervals (Cl) of AF across BW groups.
\end{abstract}

Results: During an average follow-up of 10.3 years, we identified 882 incident AF cases. LBW was associated with higher risk of AF. Compared to individuals in the medium BW category, the HR $(95 \% \mathrm{Cl})$ of AF was $1.33(0.99,1.78)$ for LBW and $1.00(0.81,1.24)$ for high BW after adjusting for sociodemographic variables ( $p$ for trend $=0.29)$. Additional adjustment for CVD risk factors did not attenuate the associations (HR 1.42, 95\% Cl 1.06, 1.90 for LBW and HR 0.86, 95\% Cl 0.69-1.07 for high BW, compared to medium BW, p for trend $=0.01$ ).

Conclusion: LBW was associated with a higher risk of AF. This association was independent of known predictors of AF and is consistent with that observed for other cardiovascular diseases.

Keywords: Atrial fibrillation, Birth weight, Race, Sex

\section{Background}

Atrial fibrillation (AF) is the most common sustained cardiac arrhythmia encountered in clinical practice [1]. AF affects more than 2 million Americans [2], increases the risk of heart failure, stroke, myocardial infarction, and overall mortality [3-6], and contributes significantly to healthcare costs [7].

The developmental origins of health and adult disease postulates that in response to a suboptimal intrauterine environment the fetus employs numerous survival mechanisms, including growth restriction, at the expense

\footnotetext{
*Correspondence: alonso@umn.edu

'Division of Epidemiology and Community Health, School of Public Health, University of Minnesota, 1300 S 2nd St, Minneapolis, MN, USA

Full list of author information is available at the end of the article
}

of an increased susceptibility to a wide range of chronic disease in adulthood. Birth weight (BW) is frequently used as a proxy for impaired fetal growth in lieu of more precise data on fetal growth trajectory. Numerous studies have suggested that low BW (LBW) is related to an increased propensity towards the development of cardiovascular $[8,9]$, metabolic, and endocrine abnormalities in later life [10].

Few data exist regarding the potential relationship between BW and subsequent risk of AF. LBW has been associated with the increased risk of development of hypertension later in life [11], an established risk factor of AF. However, the only published study that explored the impact of BW on risk of AF in adulthood, based on the Women's Health Study, found that higher BW was 
associated with an increased risk of AF, rather than decreased risk as might be expected given the fetal origins hypothesis [12].

The incidence of AF is significantly lower in African Americans compared to whites despite the increased prevalence of risk factors of AF in African Americans, such as hypertension or obesity [13-15]. In addition, the prevalence of LBW is higher among African Americans than whites [16]. Assessing whether black-white differences in BW could explain the paradoxical racial disparity in AF risk merits attention.

Therefore, the aim of this study was to assess the relationship between BW and AF risk, including assessment of effect modification by gender and race, using data collected in the Atherosclerosis Risk in Communities (ARIC) cohort.

\section{Methods}

\section{Study population}

The ARIC study is an ongoing prospective cohort in four communities in the US: Forsyth County, North Carolina; Washington County, Maryland; Minneapolis suburbs, Minnesota; and Jackson, Mississippi [17]. Participants in the cohort were randomly selected from a defined population of age 45-64. At baseline (1987-1989), 15,792 participants were recruited and received medical examinations. Three follow-up examinations were performed every three years, and a fourth follow-up exam was conducted in 2011-2013. In addition, study participants receive annual phone follow-up calls to get updates on health and vital status. The ARIC study is performed in accordance with the Declaration of Helsinki and has been approved by Institutional Review Boards (IRB) at all participating institutions: University of North Carolina at Chapel Hill IRB, Johns Hopkins University IRB, University of Mississippi Medical Center IRB, and University of Minnesota IRB. Study participants provided written informed consent at all study visits.

For this study we included only whites and African Americans who participated in ARIC visit 4 (1996-98), when BW was assessed. Individuals with missing information on BW or any other covariate of interest, reported cases of prematurity, twins, and individuals with prevalent AF at visit 4 were excluded. Table 1 shows the exclusion criteria with the resultant 10,132 observations used in the analysis.

\section{Study measures}

\section{Birth weight}

At the visit 4 exam (1996-98), participants were asked to recall their exact $\mathrm{BW}$ in pounds and ounces, and those who could not recall their exact BW were asked to categorize it into one of three categories: low $(<5.5 \mathrm{lbs} /<2.5 \mathrm{~kg}$.), medium (5.5-9.0 lbs. $/ 2.5-4.0 \mathrm{~kg})$ or high $(>9.0 \mathrm{lbs} . />4.0 \mathrm{~kg})$.
Table 1 Number of excluded participants according to different exclusion criteria, ARIC study, 1996-1998

\begin{tabular}{lll}
\hline Exclusion criteria & $\begin{array}{l}\text { Individuals } \\
\text { excluded }\end{array}$ & Participants \\
\hline Initial cohort in 1987-89 & 4,136 & 15,792 \\
Attended visit 4 (1996-98) & 298 & 11,656 \\
Prevalent AF at visit 4 & 17,358 \\
Low or bad quality ECGs at baseline & 67 & 11,183 \\
Race other than white or African American & 11,116 \\
Missing Covariates & 151 & 10,965 \\
Twin gestation or premature infant & 490 & 10,475 \\
Missing BW & 343 & 10,132 \\
\hline
\end{tabular}

Actual reported weights were grouped using these same categories. This three-level ordinal variable was used in all analyses. Participants reporting exact BW were more likely to be white and female [18]. In the ARIC cohort, categories of self-reported exact BW followed a similar distribution to BW in the general US population [18]. Studies in other populations suggest that self-reported birth weight has moderate to good validity [19].

\section{AF ascertainment}

Data on AF was obtained from 12-lead ECGs performed in study exams, from ICD-9 codes from hospitalization discharges $(427.31,427.32)$ or from death certificates if AF was listed as any cause of death (ICD-9 427.3 or ICD-10 I48) [15]. More than 90\% of AF cases were identified from hospital discharges. In the present analysis, we considered incident $\mathrm{AF}$ as any first occurrence of $\mathrm{AF}$ between visit 4 and December 31, 2008; after visit 4, all AF cases were identified from hospital discharge codes and death certificates.

\section{Other variables}

Other covariates were defined based on information collected at visit 4 (with the exception of education level and income, which were collected at visit 1). Questionnaires provided information on age, gender, educational level, income, cigarette smoking, alcohol intake, and use of hypertensive medication. Blood pressure, height and weight were measured at the study visit. Body mass index (BMI) was computed as weight in kilograms divided by height in meters squared. Diabetes was defined as fasting serum glucose $\geq 126 \mathrm{mg} / \mathrm{dL}$, use of anti-diabetic medication, non-fasting serum glucose $\geq 200 \mathrm{mg} / \mathrm{dL}$, or a self-reported diagnosis of diabetes. Prevalent MI and heart failure were defined as previously described [20,21].

\section{Statistical analysis}

The causal model guiding our selection of covariates for multivariable analysis is presented in Figure 1. A brief 


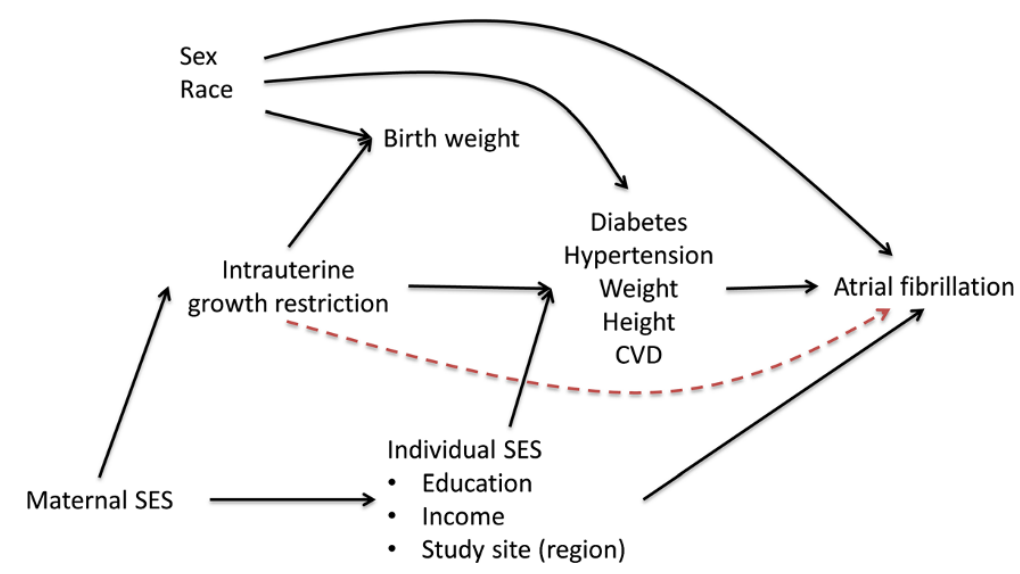

Figure 1 Causal model for the association of birth weight, as a proxy for intrauterine growth restriction, with the incidence of atrial fibrillation. The red-dashed line represents a hypothetical direct effect of intrauterine growth retardation on atrial fibrillation incidence not mediated through known cardiovascular risk factors. CVD: Cardiovascular disease. SES: Socioeconomic status.

justification for this model follows. Our main question of interest is whether intrauterine growth retardation, assessed with BW as a proxy, leads to AF. Based on existing literature, this association could be mediated by an increase in the risk of cardiovascular risk factors (diabetes, hypertension, obesity, etc.) and cardiovascular disease [8-10], which are well-established risk factors for AF [22]. Male gender and white race are associated with higher birth weight in our sample (Table 1) and are also associated with AF risk [15], therefore being considered confounders. Maternal socioeconomic status (SES), through a range of mechanisms, is associated with intrauterine growth retardation and, through its impact on an individual's SES, can affect the future risk of AF. Since we do not have information on maternal SES, we used individual SES as a proxy, including education, income, and study site as potential confounders. Finally, we considered all other cardiovascular risk factors as variables possibly in the causal pathway between intrauterine growth retardation/BW and AF risk. Estimation of the total effect of birth weight on AF should not adjust for those variables; however, a model adjusting for those variables could be used to test whether any association between $\mathrm{BW}$ and $\mathrm{AF}$ is mediated through changes in cardiovascular risk factors or through other, not described, pathways (represented by the red dashed line in Figure 1), provided that some assumptions are met. These assumption include the absence of uncontrolled confounding between the exposure and the outcome, absence of uncontrolled confounding between the mediator and the outcome, and no effect measure modification between the exposure and the mediator in their association with the outcome [23].

In all analyses, we categorized BW into low $(<2.5 \mathrm{~kg})$, medium $(2.5-4.0 \mathrm{~kg})$, and high $(>4.0 \mathrm{~kg})$. The association between categories of $\mathrm{BW}$ and incidence of $\mathrm{AF}$ was estimated using hazard ratios (HR) and 95\% confidence intervals (CI) obtained from Cox proportional hazards models, using medium BW as the reference category. An initial analysis was conducted on the entire sample, followed by race and gender-stratified analyses. Time of follow-up was defined as number of days between visit 4 and incident AF, death, lost during followup, or December 31, 2008, whichever occurred earlier. We conducted initial analysis adjusted for potential confounders: Model 1 adjusted for age, race and gender. Model 2 adjusted additionally for socioeconomic factors (income and education) and study center. Variables in models 1 and 2 can be considered confounders since they may be determinants of $\mathrm{BW}$ and are also risk factors for AF. Finally, we ran an additional model 3 that included cardiovascular risk factors as potential mediators, i.e. in the causal pathway, of the association between BW and AF risk (diabetes, systolic blood pressure (SBP), hypertension medications, smoking, height, BMI, prevalent myocardial infarction, and heart failure). Based on our assumptions about causal relationships, this model specifically tests whether a direct effect of $\mathrm{BW}$ on AF risk exists independently of these mediators (Figure 1). Assumptions for this test include: (1) no uncontrolled confounding between the exposure (BW) and the outcome (AF), (2) no uncontrolled confounding between the mediators (cardiovascular risk factors) and the outcome (AF), and (3) no effect measure modification between the exposure and the mediators [23]. We considered the first two assumptions to hold because of our adjustment for predictors of BW, AF, and cardiovascular risk factors. We did not find significant effect measure modification between BW and prevalence of cardiovascular risk factors regarding the association with AF (data not shown), considering the third assumption to hold. 
Pre-specified analyses testing for interactions by race and gender were performed including multiplicative terms in the models and conducting race- and gender-stratified analyses. Test for trends were conducted by including BW category in the models as an ordinal variable. We conducted two sensitivity analyses, first including only those participants who reported their exact BW in pounds and ounces, and second starting follow-up at ARIC visit 1, instead of visit 4, and including all incident cases from visit 1. All analyses were conducted with SAS version 9.2 (SAS Institute Inc., Cary, NC).

\section{Results}

\section{Baseline characteristics}

At visit 4, the mean age of the 10,132 eligible individuals was 62.7 years (standard deviation, 5.7). Of these, 449 (4\%) reported LBW, 1,086 (11\%) reported high BW, while the remaining 8,597 (85\%) reported medium BW.

Table 2 shows characteristics of the study sample by categories of BW. Approximately $44 \%$ of the eligible participants were men. Women and African Americans were more likely to be in the LBW category $(\mathrm{P}<0.001)$.

\section{Association between BW category and incident AF}

During an average 10.3 years of follow-up, we identified 882 incident $\mathrm{AF}$ cases. The crude incidence rate of $\mathrm{AF}$ in the study population was 8.4 events per 1000 person years. Incidence rates across BW categories were: 10.5 events per 1000 person-years for those with LBW, 8.3 events per 1000 person-years for the medium BW category, and 8.8 events per 1000 person-years for the high BW category (Table 3).
In multivariable models, LBW was associated with a higher risk of AF compared to medium BW (Table 3). After adjusting for socio-demographic variables (Model 2), individuals in the LBW category had an approximate 33\% increase in AF risk compared to those with medium BW: HR 1.33, 95\% CI 0.99, 1.78. High BW, compared to medium BW, was not associated with the risk of AF (HR 1.00, 95\% CI 0.81, 1.24). The association remained after controlling for potential mediators (Model 3), suggesting the presence of alternative pathways between $\mathrm{BW}$ and AF risk. Results remained essentially unchanged when we started the follow-up at visit 1, including all incident cases occurring afterwards (1131 AF cases; Model 2: HR 1.34, 95\% CI 1.03, 1.74 for LBW; HR 1.04, 95\% CI 0.86, 1.25 for high $\mathrm{BW}$, compared to medium $\mathrm{BW}$ ).

An analysis restricted to the 4810 ARIC participants (including 412 AF cases) who reported their exact BW in pounds and ounces provided similar estimates of association. The HRs (95\% CI) for low and high BW compared to medium BW were respectively $1.23(0.85$, $1.79)$ and $0.91(0.71,1.16)$ in models adjusted for sociodemographic variables, and $1.32(0.91,1.93)$ and 0.81 $(0.63,1.04)$ after additional adjustment for cardiovascular disease risk factors.

\section{Association between $\mathrm{BW}$ and incident $\mathrm{AF}$ by race}

The interaction term race"BW was not statistically significant ( $\mathrm{p}$-value $=0.28$ ). Nonetheless, we conducted racestratified analysis, as pre-specified in our hypothesis. Table 4 shows the association between BW and AF by race. Among whites, LBW was associated with higher risk of AF, with an increase of 35\% in AF risk in models

Table 2 Baseline characteristics of study participants by self-reported birth weight categories, ARIC study, 1996-2008

\begin{tabular}{|c|c|c|c|c|}
\hline & \multicolumn{3}{|c|}{ Birth weight categories } & \multirow[b]{2}{*}{ P value } \\
\hline & Low & Medium & High & \\
\hline $\mathrm{N}(\%)$ & $449(4 \%)$ & $8,597(85 \%)$ & $1,086(11 \%)$ & \\
\hline Age, years (mean $\pm S D$ ) & $62.7 \pm 5.6$ & $62.7 \pm 5.7$ & $62.8 \pm 5.6$ & 0.89 \\
\hline Male, \% & 22.1 & 42.6 & 59.1 & $<0.001$ \\
\hline African American, \% & 26.7 & 21.1 & 16.3 & $<0.001$ \\
\hline Less than high school degree, \% & 25.2 & 18.3 & 15.8 & $<0.001$ \\
\hline BMI, kg/m² (mean $\pm \mathrm{SD})$ & $28.5 \pm 6.0$ & $28.7 \pm 5.5$ & $29.4 \pm 5.9$ & 0.001 \\
\hline Height, cm (mean \pm SD) & $162.6 \pm 8.6$ & $168.2 \pm 9.0$ & $172.5 \pm 9.1$ & $<0.001$ \\
\hline Diabetes, \% & 19.8 & 15.8 & 14.6 & 0.04 \\
\hline $\mathrm{SBP}, \mathrm{mmHg}($ mean $\pm \mathrm{SD})$ & $129.3 \pm 19.1$ & $127.4 \pm 18.9$ & $125.4 \pm 18.0$ & $<0.001$ \\
\hline Hypertensive medication use, \% & 47.4 & 42.4 & 39.2 & 0.01 \\
\hline Current smoker, \% & 17.8 & 14.2 & 17.3 & $<0.001$ \\
\hline Prevalent heart failure, \% & 1.3 & 1.3 & 2.2 & 0.06 \\
\hline Prevalent myocardial infarction, \% & 3.3 & 2.7 & 2.9 & 0.69 \\
\hline Incident atrial fibrillation, \% & 10.9 & 8.6 & 9.2 & 0.21 \\
\hline
\end{tabular}


Table 3 Hazard ratios and 95\% confidence intervals of atrial fibrillation by birth weight category, ARIC study, 19962008

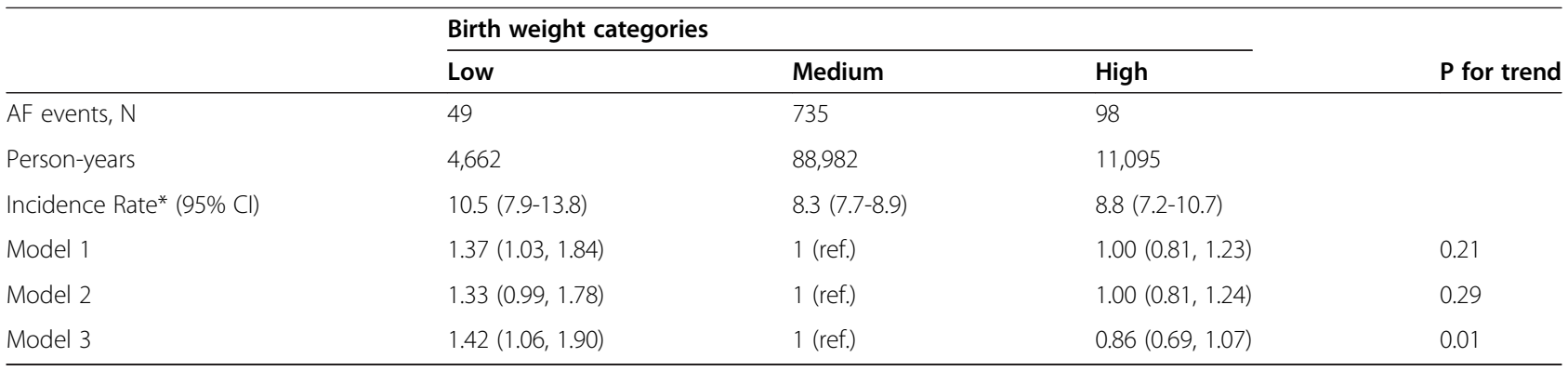

*Incidence rate per 1000 person years.

Model 1: Cox proportional hazards model adjusted for age, race and gender.

Model 2: As model 1, additionally adjusted for study center, income, and education.

Model 3: As model 2, additionally adjusted for diabetes, systolic blood pressure, hypertension medications, smoking, height, body mass index, prevalent. myocardial infarction and heart failure.

adjusted for sociodemographic characteristics, compared to LBW. The association was less apparent among African Americans, with individuals in the medium BW category showing the lowest risk of AF. AfricanAmerican race was associated with lower risk of AF compared to white race after adjusting for age, gender, and birth weight category (HR 0.84, 95\% CI 0.70-1.00).

\section{Association between BW category and incident AF by gender}

As with race, no significant interaction was observed between gender and BW ( $\mathrm{p}$-value $=0.21$ ). The pre-specified analysis showing the association between $\mathrm{BW}$ and AF risk by gender is presented in Table 5 . LBW showed a strong association with AF risk in men, while in women, no clear evidence of an association between BW and AF risk was present.

\section{Discussion}

Our study showed an association between BW and incident AF, with higher risk of AF among individuals with LBW, compared to medium BW. These results are similar to what has been generally observed for the relationship of BW with other cardiovascular diseases $[8,9]$. The association was more evident in men and whites; BW was a weaker risk factor for AF in women and African Americans. Our results are consistent with the fetal origin hypothesis of coronary heart disease

Table 4 Hazard ratios and $95 \%$ confidence intervals of atrial fibrillation by birth weight category, stratified by race, ARIC study, 1996-2008

\begin{tabular}{|c|c|c|c|c|}
\hline \multirow[b]{2}{*}{ Whites $(N=8,022, A F$ events $=743)$} & \multicolumn{3}{|c|}{ Birth weight categories } & \multirow[b]{2}{*}{ P for trend } \\
\hline & Low & Medium & High & \\
\hline AF events, $\mathrm{N}$ & 38 & 624 & 81 & \\
\hline Person-years & 3,471 & 70,183 & 9,304 & \\
\hline Incidence Rate* (95\% Cl) & $10.9(7.9-14.9)$ & $8.9(8.2-9.6)$ & $8.7(7.0-10.8)$ & \\
\hline Model 1 & $1.35(0.97,1.88)$ & 1 (ref.) & $0.92(0.73,1.16)$ & 0.10 \\
\hline Model 2 & $1.30(0.94,1.81)$ & 1 (ref.) & $0.92(0.73,1.16)$ & 0.13 \\
\hline Model 3 & $1.38(0.99,1.92)$ & 1 (ref.) & $0.79(0.63,1.00)$ & 0.007 \\
\hline African Americans $(\mathrm{N}=2,110, \mathrm{AF}$ events $=139)$ & Low & Medium & High & \\
\hline AF events, $N$ & 11 & 111 & 17 & \\
\hline Person-years & 1,191 & 18,798 & 1,791 & \\
\hline Incidence Rate* (95\% Cl) & $9.2(4.9-16.0)$ & $5.9(4.9-7.1)$ & $9.5(5.7-14.9)$ & \\
\hline Model 1 & $1.51(0.81,2.81)$ & 1 (ref.) & $1.64(0.98,2.74)$ & 0.55 \\
\hline Model 2 & $1.49(0.80,2.78)$ & 1 (ref.) & $1.75(1.04,2.93)$ & 0.44 \\
\hline Model 3 & $1.60(0.86,2.99)$ & 1 (ref.) & $1.37(0.80,2.34)$ & 0.97 \\
\hline
\end{tabular}

*Incidence rate per 1000 person years.

Model 1: Cox proportional hazards model adjusted for age and gender.

Model 2: As model 1, additionally adjusted for study center, income, and education.

Model 3: As model 2, additionally adjusted for diabetes, systolic blood pressure, hypertension medications, smoking, height, body mass index, prevalent.

myocardial infarction and heart failure. 
and other developmental theories that associate cardiovascular disease in adulthood with undernutrition in utero [24]. Adverse prenatal factors that may have caused intrauterine growth restriction could also lead to metabolic and organ changes that can predispose affected individuals to chronic disease in adulthood. This suggests that LBW, an indication of possible intrauterine growth restriction, may be a risk factor for developing AF later in life.

LBW has been associated with a higher risk for hypertension and diabetes $[10,11]$. Reduced fetal growth may result in changes in fetal blood flow or hormonal variations, which can lead to abnormal development of various organs involved in blood pressure control including the kidneys, autonomic nervous system, endocrine glands, and cardiac vasculature. In the kidney, these changes would result in fewer nephrons, potentially leading to poor salt regulation and hypertension [25]. In the pancreas, they could result in a reduced beta cell mass and poor regulation of glucose later in life predisposing one to diabetes. It has been suggested that the higher prevalence of diabetes in individuals with LBW could reflect a selective survival of LBW infants genetically susceptible to diabetes [26]. Studies have shown that obesity, diabetes and hypertension are strong risk factors for developing AF [27,28]. Therefore, the increased risk of AF associated with LBW could be mediated through a higher risk of cardiometabolic risk factors. In our analysis, however, associations remained after adjustment for cardiovascular risk factors, which would suggest that other variables mediate this association.
Results from the ARIC Study contradict those previously reported in the Women's Health Study, which found a higher risk of AF among women with higher BW [12]. Some important differences exist between both studies, though. The Women's Health Study included only women, mostly white, all of them health professionals. The ARIC study, in contrast, included a biracial population of both genders, with a more diverse educational background. Other differences between studies include the potential different quality of BW information (possibly higher in the Women's Health Study, since participants were all female and more educated on average), the method for ascertainment of AF, and the availability of potential confounders. Future research in other population should try to clarify this apparent inconsistency.

In race and gender-stratified models, we found that the association of LBW with the risk of incident AF was stronger in men and whites than in women and African Americans, although these differences were not statistically significant. Possible explanations for the gender and race disparity are the differential distribution of BW and different degree of misclassification in BW information across different demographic groups. Of note, the previously reported lower AF risk in African Americans vs whites [15] in the ARIC cohort remained after adjustment for birth weight categories.

\section{Study limitations and strengths}

Several important limitations exist regarding the information on BW. First, about $50 \%$ of the participants

Table 5 Hazard ratio and $95 \%$ confidence intervals of atrial fibrillation by birth weight category, stratified by gender, ARIC study, 1996-2008

\begin{tabular}{|c|c|c|c|c|}
\hline \multirow[b]{2}{*}{ Men $(N=4,403$, AF events $=453)$} & \multicolumn{3}{|c|}{ Birth weight categories } & \multirow[b]{2}{*}{$P$ for trend } \\
\hline & Low & Medium & High & \\
\hline AF events, $N$ & 19 & 374 & 60 & \\
\hline Person-years & 982 & 36,754 & 6,410 & \\
\hline Incidence Rate* (95\% CI) & $19.4(12.0-29.6)$ & $10.2(9.2-11.2)$ & $9.4(7.2-12.0)$ & \\
\hline Model 1 & $1.92(1.21,3.04)$ & 1 (ref.) & $0.92(0.70,1.20)$ & 0.07 \\
\hline Model 2 & $1.91(1.20,3.03)$ & 1 (ref.) & $0.92(0.70,1.20)$ & 0.07 \\
\hline Model 3 & $1.91(1.20,3.04)$ & 1 (ref.) & $0.80(0.61,1.06)$ & 0.009 \\
\hline Women $(\mathrm{N}=5,729, \mathrm{AF}$ events $=429)$ & Low & Medium & High & \\
\hline AF events, $N$ & 30 & 361 & 38 & \\
\hline Person-years & 3,680 & 52,227 & 4,685 & \\
\hline Incidence Rate* (95\% Cl) & $8.2(5.6-11.5)$ & $6.9(6.2-7.7)$ & $8.1(5.8-11.0)$ & \\
\hline Model 1 & $1.17(0.80,1.69)$ & 1 (ref.) & $1.16(0.83,1.62)$ & 0.92 \\
\hline Model 2 & $1.13(0.78,1.64)$ & 1 (ref.) & $1.17(0.83,1.63)$ & 0.80 \\
\hline Model 3 & $1.23(0.84,1.79)$ & 1 (ref.) & $0.97(0.67,1.36)$ & 0.51 \\
\hline
\end{tabular}

*Incidence rate per 1000 person years.

Model 1: Cox proportional hazards model adjusted for age and gender.

Model 2: As model 1, additionally adjusted for study center, income, and education.

Model 3: As model 2, additionally adjusted for diabetes, systolic blood pressure, hypertension medications, smoking, height, body mass index, prevalent.

myocardial infarction and heart failure. 
could not recall their exact BW and, thus, only BW categories could be used (low, medium, high BW). Second, the overall prevalence of LBW in our sample $(4 \%)$ is lower than expected, suggesting underreporting of LBW [18]. In an analysis including only those with selfreported exact BW, which possibly provided more valid BW information [18], results remained unchanged. Third, the absence of gestational age information also adds to misclassification errors; infants experiencing normal fetal growth but born somewhat earlier are combined with those that are small for gestational age, which suggests intrauterine growth restriction. However, participants were asked if they were born premature, and excluded if the answer was affirmative, thus decreasing the magnitude of this potential misclassification. Similarly, the relatively low number of AF events in the LBW category may compromise the robustness of our results. Another important limitation is that AF was mostly ascertained from hospital discharge codes, which could lead to missing AF events identified in outpatient settings only. We and others have showed previously, however, that this method has adequate validity for epidemiologic studies of AF [15,29]. Finally, BW information was not collected until study participants were in late midlife (average age at baseline 63), which might lead to selection bias if study dropout status or non-participation was associated with BW and AF or AF risk factors. However, we adjusted our analysis for known established risk factors for AF thus reducing this bias [30].

Major strengths of this study include the large sample size, the inclusion of both whites and African Americans, extended follow-up, and availability of information on confounding variables (socioeconomic status) and mediators (anthropometry, cardiovascular risk factors).

\section{Conclusion}

In conclusion, in this biracial cohort study, LBW was associated with an increased risk of incident AF. This association was independent of known predictors of AF such as hypertension, BMI and height. This association may be stronger in men and whites. Given the differences in results from the ARIC Study and the Women's Health Study, additional research in other diverse populations should be conducted to clarify the relationship between birth weight and AF risk.

The authors thank the staff and participants of the ARIC study for their important contributions.

\section{Competing interests}

The authors have no conflict of interest to disclose related to the present manuscript.

\section{Authors' contributions}

The study was designed by SOW, EWD, KMR, and AA. Data on AF were collected by EZS and AA. SOW and FLL analyzed the data. SOW and AA wrote the first draft of the manuscript. All authors participated in data interpretation, discussion and preparation of the final manuscript, and read and approved the final manuscript.

\section{Acknowledgments}

The Atherosclerosis Risk in Communities Study is carried out as a collaborative study supported by National Heart, Lung, and Blood Institute contracts (HHSN268201100005C, HHSN268201100006C,

HHSN268201100007C, HHSN268201100008C, HHSN268201100009C, HHSN268201100010C, HHSN268201100011C, and HHSN268201100012C). This study was additionally supported by grants RC1-HL099452 from the National Heart, Lung, and Blood Institute and 09SDG2280087 from the American Heart Association.

\section{Author details}

'Division of Epidemiology and Community Health, School of Public Health, University of Minnesota, 1300 S 2nd St, Minneapolis, MN, USA.

${ }^{2}$ Epidemiological Cardiology Research Center (EPICARE), Wake Forest School of Medicine, Winston-Salem, NC, USA. ${ }^{3}$ School of Population Health, University of Queensland, Brisbane, Queensland, Australia. ${ }^{4}$ Department of Epidemiology, University of North Carolina, Chapel Hill, NC, USA, and Social and Scientific Systems, Inc, Durham, NC, USA.

Received: 21 February 2014 Accepted: 23 May 2014

Published: 26 May 2014

\section{References}

1. Magnani JW, Rienstra M, Lin H, Sinner MF, Lubitz SA, McManus DD, Dupuis J, Ellinor PT, Benjamin EJ: Atrial fibrillation: current knowledge and future directions in epidemiology and genomics. Circulation 2011, 124:1982-1993.

2. Go AS, Hylek EM, Phillips KA, Chang YC, Henault LE, Selby JV, Singer DE: Prevalence of diagnosed atrial fibrillation in adults. National implications for rhythm management and stroke prevention: the anticoagulation and risk factors in atrial fibrillation (ATRIA) study. JAMA 2001, 285:2370-2375.

3. Wolf PA, Abbott RD, Kannel WB: Atrial fibrillation as an independent risk factor for stroke: the Framingham study. Stroke 1991, 22:983-988.

4. Benjamin EJ, Wolf PA, D'Agostino RB, Silbershatz H, Kannel WB, Levy D: Impact of atrial fibrillation on the risk of death: the Framingham heart study. Circulation 1998, 98:946-952.

5. Conen D, Chae CU, Glynn RJ, Tedrow UB, Everett BM, Buring JE, Albert CM: Risk of death and cardiovascular events in initially healthy women with new-onset atrial fibrillation. JAMA 2011, 305:2080-2087.

6. Soliman EZ, Safford MM, Muntner P, Khodneva Y, Dawood FZ, Zakai NA, Thacker EL, Judd S, Howard VJ, Howard G, Herrington DM, Cushman M: Atrial fibrillation and the risk of myocardial infarction. JAMA Intern Med 2014, 174:107-114.

7. Kim MH, Johnston SS, Chu B-C, Dalal MR, Schulman KL: Estimation of total incremental health care costs in patients with atrial fibrillation in the United States. Circ Cardiovasc Qual Outcomes 2011, 4:313-320.

8. Huxley R, Owen CG, Whincup PH, Cook DG, Rich-Edwards J, Smith GD, Collins R: Is birth weight a risk factor for ischemic heart disease in later life? Am J Clin Nutr 2007, 85:1244-1250.

9. Eriksson JG, Forsén T, Tuomilehto J, Osmond C, Barker DJP: Early growth, adult income, and risk of stroke. Stroke 2000, 31:869-874.

10. Whincup PH, Kaye SJ, Owen CG, Huxley RR, Cook DG, Anazawa S, BarrettConnor E, Bhargava SK, Birgisdottir BE, Carlsson S, de Rooij SR, Dyck RF, Eriksson JG, Falkner B, Fall C, Forsén T, Grill V, Gudnason V, Hulman S, Hypponen E, Jeffreys M, Lawlor DA, Leon DA, Minami J, Mishra G, Osmond C, Power C, Rich-Edwards JW, Roseboom TJ, Sachdev HS, et al: Birth weight and risk of type 2 diabetes: a systematic review. JAMA 2008, 300:2886-2897.

11. Huxley RR, Shiell AW, Law CM: The role of size at birth and postnatal catch-up growth in determining systolic blood pressure: a systematic review of the literature. J Hypertens 2000, 18:815-831.

12. Conen D, Tedrow UB, Cook NR, Buring JE, Albert CM: Birth weight is a significant risk factor for incident atrial fibrillation. Circulation 2010, 122:764-770.

13. Soliman EZ, Alonso A, Goff DC Jr: Atrial fibrillation and ethnicity: the known, the unknown and the paradox. Future Cardiol 2009, 5:547-556.

14. Psaty BM, Manolio TA, Kuller LH, Kronmal RA, Cushman M, Fried LP, White R, Furberg C, Rautaharju PM: Incidence of and risk factors for atrial fibrillation in older adults. Circulation 1997, 96:2455-2461. 
15. Alonso A, Agarwal SK, Soliman EZ, Ambrose M, Chamberlain AM, Prineas RJ, Folsom AR: Incidence of atrial fibrillation in whites and African-Americans: the atherosclerosis risk in communities (ARIC) study. Am Heart J 2009, 158:111-117.

16. David RJ, Collins JW: Differing birth weight among infants of U.S.-born blacks, African-born blacks, and U.S.-born whites. N Engl J Med 1997, 337:1209-1214.

17. The ARIC Investigators: The atherosclerosis risk in communities (ARIC) study: design and objectives. Am J Epidemiol 1989, 129:687-702.

18. Rose KM, Tilling K, Folsom AR, Coresh J: Evaluation of self-reported birth weight in older adults. Circulation 2004, 7:E127-E127.

19. Tehranifar P, Liao Y, Flom JD, Terry MB: Validity of self-reported birth weight by adult women: sociodemographic influences and implications for life-course studies. Am J Epidemiol 2009, 170:910-917.

20. White AD, Folsom AR, Chambless LE, Sharret AR, Yang K, Conwill D, Higgins M, Williams OD, Tyroler HA, The ARIC Investigators: Community surveillance of coronary heart disease in the atherosclerosis risk in communities (ARIC) study: methods and initial two years' experience. J Clin Epidemiol 1996, 49:223-233.

21. Loehr LR, Rosamond WD, Chang PP, Folsom AR, Chambless LE: Heart failure incidence and survival (from the atherosclerosis risk in communities study). Am J Cardiol 2008, 101:1016-1022.

22. Alonso A, Krijthe BP, Aspelund T, Stepas KA, Pencina MJ, Moser CB, Sinner MF, Sotoodehnia N, Fontes JD, Janssens ACJW, Kronmal RA, Magnani JW, Witteman JC, Chamberlain AM, Lubitz SA, Schnabel RB, Agarwal SK, McManus DD, Ellinor PT, Larson MG, Burke GL, Launer $\mathrm{L}$, Hofman A, Levy D, Gottdiener JS, KÃøÃøb S, Couper D, Harris TB, Soliman EZ, Stricker BHC, et al: Simple risk model predicts incidence of atrial fibrillation in a racially and geographically diverse population: the CHARGE-AF Consortium. J Am Heart Assoc 2013, 2:e000102.

23. Kaufman JS, MacLehose RF, Kaufman S: A further critique of the analytic strategy of adjusting for covariates to identify biologic mediation. Epidemiol Perspect Innov 2004, 1:4.

24. Barker DJP: The developmental origins of adult disease. J Am Coll Nutr 2004, 23:588S-595S.

25. Brenner BM, Chertow GM: Congenital oligonephropathy and the etiology of adult hypertension and progressive renal injury. Am J Kidney Dis 1994, 23:171-175

26. Hales CN, Barker DJP: The thrifty phenotype hypothesis. Br Med Bull 2001, 60:5-20.

27. Magnani JW, Hylek EM, Apovian CM: Obesity begets atrial fibrillation: a contemporary summary. Circulation 2013, 138:401-405.

28. Huxley RR, Lopez FL, Folsom AR, Agarwal SK, Loehr LR, Soliman EZ, Maclehose R, Konety S, Alonso A: Absolute and attributable risks of atrial fibrillation in relation to optimal and borderline risk factors: the atherosclerosis risk in communities (ARIC) study. Circulation 2011, 123:1501-1508.

29. Jensen PN, Johnson K, Floyd J, Heckbert SR, Carnahan R, Dublin S: A systematic review of validated methods for identifying atrial fibrillation using administrative data. Pharmacoepidemiol Drug Saf 2012, 21(Suppl 1):141-147.

30. Hernán MA, Hernández-Díaz S, Robins JM: A structural approach to selection bias. Epidemiology 2004, 15:615-625.

doi:10.1186/1471-2261-14-69

Cite this article as: Lawani et al:: Birth weight and the risk of atrial

fibrillation in whites and African Americans: the Atherosclerosis Risk In Communities (ARIC) study. BMC Cardiovascular Disorders 2014 14:69.

\section{Submit your next manuscript to BioMed Central and take full advantage of:}

- Convenient online submission

- Thorough peer review

- No space constraints or color figure charges

- Immediate publication on acceptance

- Inclusion in PubMed, CAS, Scopus and Google Scholar

- Research which is freely available for redistribution

Submit your manuscript at www.biomedcentral.com/submit
C Biomed Central 